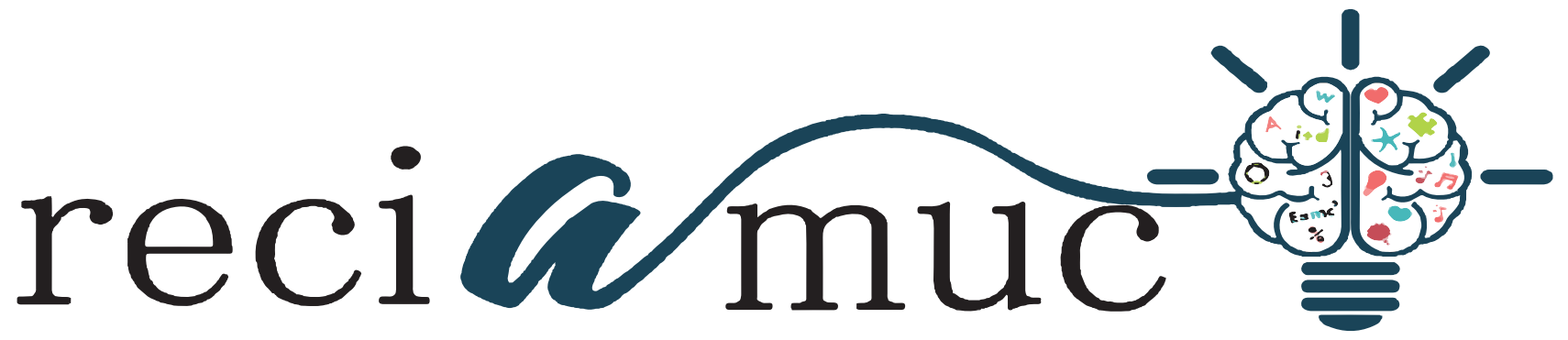

DOI: 10.26820/reciamuc/4.(4).diciembre.2020.35-46

URL: https://reciamuc.com/index. php/RECIAMUC/article/view/570

EDITORIAL: Saberes del Conocimiento

REVISTA: RECIAMUC

ISSN: 2588-0748

TIPO DE INVESTIGACIÓN: Artículo de Revisión

Código UNESCO: 32 Ciencias Médicas

PAGINAS: $35-46$

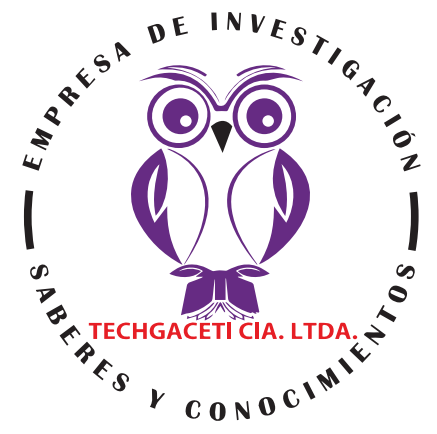

\title{
Valoración mediante ECOFAST en trauma de tórax
}

ECOFAST assessment in chest trauma

Avaliação ECOFAST em trauma torácico

\section{Melissa Fernanda Mera Cáceres'; Diana Katherine Guachamín Abril2; Toa Natali Paca Ajitimbay³; Darlys Tatiana Cerón ${ }^{4}$}

\section{RECIBIDO: 23/10/2020 ACEPTADO: 17/11/2020 PUBLICADO: 24/12/2020}

1. Médico residente asistencial, Hospital Militar Quito; Ecuador; melissameracaceres@gmail.com; (D) https://orcid.org/0000-0003-3969-3521

2. Medico General; Investigador Independiente; Quito; Ecuador; dianaguachaminabril@gmail.com; (D) https:// orcid.org/0000-0002-6406-7314

3. Medico residente de Medicina Interna; Riobamba, Ecuador; natalipaca@yahoo.com (D) https://orcid.org/00000001-5146-4006

4. Médico asistencial Unidad de cuidados intensivos; Popayàn, Colombia; taticeron18@gmail.com; (D) https:// orcid.org/0000-0001-8454-0968

\section{CORRESPONDENCIA}

Melissa Fernanda Mera Cáceres

melissameracaceres@gmail.com

\section{Quito, Ecuador}




\section{RESUMEN}

El trauma es la principal causa de muerte en personas menores de 45 años en y responsable de un tercio de todos los ingresos a la unidad de cuidados intensivos. El conocimiento cada vez mayor sobre los métodos de diagnóstico por ultrasonido y su disponibilidad para lesiones potencialmente mortales como el taponamiento cardíaco y la rotura de órganos sólidos de la cavidad abdominal, que conduce a un shock hemorrágico, el diagnóstico y el monitoreo, conducen al desarrollo de FAST (Evaluación enfocada con ecografía para traumatismos) protocolo, destinado a ser utilizado tanto en el ámbito de la unidad de cuidados intensivos como de emergencia. Debido a su reproducibilidad, falta de exposición a la radiación y viabilidad al lado de la cama, esta tecnología se acepta cada vez más. Una nueva extensión del protocolo, el Extended-FAST (eFAST), proporciona información valiosa para mejorar el manejo de los pacientes, extendiendo su disponibilidad desde las afecciones abdominales a otros diagnósticos como hemotórax, derrame pleural y neumotórax. Se debe subrayar que esta técnica es capaz de reemplazar la tomografía computarizada y el diagnóstico de lavado peritoneal, y no retrasar el procedimiento quirúrgico en lugar de realizar este examen. Por lo tanto, su valoración cuidadosa en relación con la información clínica debe orientar los enfoques terapéuticos, especialmente en lugares inhóspitos como unidades de cuidados intensivos en zonas de guerra, lugares rurales o distantes, donde otros métodos de imagenología no están disponibles.

Palabras clave: Ecografía, Trauma; Cuidados intensivos, Sistemas en el punto de atención.

\section{ABSTRACT}

Trauma is the leading cause of death in people under the age of 45 in and responsible for one-third of all admissions to the intensive care unit. Increasing knowledge about ultrasound diagnostic methods and their availability for life-threatening injuries such as cardiac tamponade and rupture of solid organs of the abdominal cavity, leading to hemorrhagic shock, diagnosis and monitoring, lead to the development of the FAST (Focused Assessment with Ultrasound for Trauma) protocol, intended to be used in both the intensive care unit and emergency settings. Due to its reproducibility, lack of radiation exposure, and bedside feasibility, this technology is becoming increasingly accepted. A new protocol extension, Extended-FAST (eFAST), provides valuable information to improve patient management, extending its availability from abdominal conditions to other diagnoses such as hemothorax, pleural effusion and pneumothorax. It should be emphasized that this technique is capable of replacing computed tomography and peritoneal lavage diagnosis, and not delaying the surgical procedure instead of performing this examination. Therefore, its careful assessment in relation to clinical information should guide therapeutic approaches, especially in inhospitable settings such as intensive care units in war zones, rural or distant locations, where other imaging methods are not available.

Keywords: Ultrasound, Trauma; Intensive care, Systems at the point of care.

\section{RESUMO}

A hidronefrose congênita (Primária ou Fetal) está localizada dentro de um amplo espectro de anomalias congênitas do rim e do trato urinário que são comumente identificadas no período pré-natal. Sua origem vem de um defeito no desenvolvimento do sistema urinário em diferentes níveis, ou seja, do sistema coletor, do parênquima renal ou da migração. $\mathrm{O}$ objetivo que se pretendia alcançar era resumir diferentes critérios de especialista em relação à classificação da hidronefrose congênita. A pesquisa foi desenvolvida com base em um desenho bibliográfico e uma metodologia de revisão, com a qual foi exposta sobre: definições fundamentais, etiologia e classificação em uso. Em conclusão, a utilização da classificação mais recente proposta através do consenso realizado em 2014 entre várias sociedades médicas, a maioria dos EUA, porque permite estudar a relação entre vários graus de dilatação do trato urinário e resultados clinicamente relevantes, além de favorecer a detecção oportuna de certos casos que, em particular, poderiam levar a complicações durante o período pós-natal.

Palavras-chave: Ultrasom, Trauma; Cuidados intensivos, Sistemas no ponto de atendimento. 


\section{Introducción}

El uso de la ecografía en pacientes politraumatizados, especialmente según el protocolo FAST (Focused Assessment with Sonography for Trauma), no se limita al paciente inicialmente estable o inestable, la evaluación esa demás es una herramienta valiosa para el seguimiento. Teniendo en cuenta que estar estable no significa necesariamente estar libre de amenazas a la vida, pero, dependiendo de la mecánica del trauma puede ser una condición potencialmente peligrosa, en este caso "Ios dispositivos portátiles que proporcionan una identificación temprana de lesiones que amenazan la vida, en consecuencia, cambian su curso natural, y pueden convertirse en vida decididores y muerte" (CDC, 2004).

Un ejemplo práctico podría incluir un paciente con traumatismo abdominal cerrado sin signos clínicos de taponamiento cardía$c o$, en quien la ecografía temprana identifica signos de derrame pericárdico con restricción miocárdica, lo que cambiaría radicalmente el enfoque de una intervención inmediata. Los autores Lichtenstein, Pinsky, \& Jardin, (2002) exponen que "se desarrolló una extensión del protocolo FAST, Extended-FAST, con el objetivo de extender la evaluación, previamente restringida a las evaluaciones del corazón y de la pared abdominal, a la cavidad torácica, permitiendo el diagnóstico de neumotórax, hemotórax y ruptura diafragmática".

Mediante el siguiente cuadro se describen las principales indicaciones de eFAST

\begin{tabular}{|l|}
\hline \multicolumn{1}{|c|}{ eFAST } \\
\hline Traumatismo cardíaco \\
penetrante \\
Traumatismo cardíaco \\
cerrado Traumatismo \\
abdominal cerrado \\
Traumatismo torácico \\
Neumotórax \\
Hemotórax \\
Hipotensión de causa indefinida \\
\hline
\end{tabular}

Cuadro 1. Indicaciones de EFAST

Fuente: (Lichtenstein, Pinsky, \& Jardin, 2002).

En tal sentido, cuando existe un panorama de un paciente "Estable" debe significar "mantenerse alerta y vigilar al paciente; por otro lado, la inestabilidad debe entenderse como una condición clínica en la que se requieren medidas inmediatas para evitar resultados catastróficos. Años anteriores los autores Peitzman, Billiar, Harbrecht, \& Kelly, (1995) evaluaron "la etiología del shock por trauma cerrado, y describió como causa primaria frecuencia decreciente, la hipovolemia $(59 \%)$, el traumatismo craneoencefálico (16\%), la obstrucción neumotórax, taponamiento cardíaco (13\%), shock neurogénico $(7 \%)$ y otros $(7 \%) "$.

La identificación del sitio de sangrado y su control es vital, ya que las medidas de reanimación, como las infusiones de cristaloides, pueden tener una respuesta transitoria o nula si el sitio de sangrado no se trata. En caso de lesión vascular, el tratamiento definitivo es vital, al igual que la terapia de control de daños en casos seleccionados. De acuerdo a la importancia de un diagnostico acertado y a una valoración lo antes posible en pacientes que presentan traumatismo, se tiene como objetivo principal desarrollar una investigación que brinde información de interés a lectores del área, dando a conocer los beneficios de este tipo de opciones de imagenología para ayudar

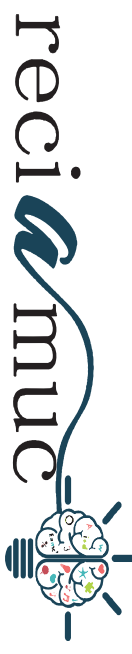


a los pacientes en estado crítico y ofrecer el abordaje necesario.

\section{Metodología}

Para el desarrollo de este proceso investigativo, se plantea como metodología la encaminada hacia una orientación científica particular que se encuentra determinada por la necesidad de indagar en forma precisa y coherente una situación, en tal sentido Davila, (2015) define la metodología "como aquellos pasos anteriores que son seleccionados por el investigador para lograr resultados favorables que le ayuden a plantear nuevas ideas" (p.66)

Lo citado por el autor, lleva a entender que el desarrollo de la acción investigativa busca simplemente coordinar acciones enmarcadas en una revisión bibliográfica con el fin de complementar ideas previas relacionadas Valoración mediante ecofast en trauma de tórax a través de una revisión de literatura, para así finalmente elaborar un cuerpo de consideraciones generales que ayuden a ampliar el interés propuesto.

\section{Tipo de Investigación}

Dentro de toda práctica investigativa, se precisan acciones de carácter metodológico mediante las cuales se logra conocer y proyectar los eventos posibles que la determinan. En este sentido, la presente investigación corresponde al tipo documental, definido por Castro (2016), "se ocupa del estudio de problemas planteados a nivel teórico, la información requerida para abordarlos se encuentra básicamente en materiales impresos, audiovisuales y / o electrónicos". (p.41).

En consideración a esta definición, la orientación metodológica incluye la oportunidad de cumplir con una serie de actividades inherentes a la revisión y lectura de diversos documentos, donde se encuentran ideas explicitas relacionadas con los tópicos encargados de identificar una característica inmersa en el estudio. Por lo tanto, se rea- lizaron continuas interpretaciones con el claro propósito de revisar aquellas apreciaciones propuestas por diferentes investigadores en relación al tema de interés, para luego dar la respectiva argumentación a los planteamientos, en función a las necesidades encontradas en la investigación, apoyados en las herramientas tecnológicas para la búsqueda de trabajos con valor científico disponibles en la web que tenían conexión con el objetivo principal de la investigación.

\section{Fuentes Documentales}

El análisis correspondiente a las características que predomina en el tema seleccionado, llevan a incluir diferentes fuentes documentales encargadas de darle el respectivo valor científico y en ese sentido cumplir con la valoración de los hechos a fin de generar nuevos criterios que sirven de referencia a otros procesos investigativos. Para Castro,(2016) las fuentes documentales incorporadas en la investigación documental o bibliográfica, "representa la suma de materiales sistemáticos que son revisados en forma rigurosa y profunda para llegar a un análisis del fenómeno" (p.41). Por lo tanto, se procedió a cumplir con la lectura previa determinada para encontrar aquellos aspectos estrechamente vinculados con el tema, con el fin de explicar mediante un desarrollo las respectivas apreciaciones generales de importancia.

\section{Técnicas para la Recolección de la Infor- mación}

La conducción de la investigación para ser realizada en función a las particularidades que determinan a los estudios documentales, tiene como fin el desarrollo de un conjunto de acciones encargadas de llevar a la selección de técnicas estrechamente vinculadas con las características del estudio. Bolívar, (2015), refiere, que es "una técnica particular para aportar ayuda a los procedimientos de selección de las ideas primarias y secundarias". (p.71). 
Tal como lo expresa, Bolívar, (2015) "Las técnicas documentales proporcionan las herramientas esenciales y determinantes para responder a los objetivos formulados y llegar a resultados efectivos" (p. 58). Es decir, para responder con eficiencia a las necesidades investigativas, se introdujeron como técnica de recolección el método inductivo, que hizo posible llevar a cabo una valoración de los hechos de forma particular para llegar a la explicación desde una visión general. El autor Bolívar, (2015) tambien expresa que las técnicas de procesamiento de datos en los estudios documentales "son las encargadas de ofrecer al investigador la visión o pasos que deben cumplir durante su ejercicio, cada una de ellas debe estar en correspondencia con el nivel a emplear" (p. 123). Esto indica, que para llevar a cabo el procesamiento de los datos obtenidos una vez aplicadas las técnicas seleccionadas, tales como: fichas de resumen, textual, registros descriptivos entre otros, los mismos se deben ajustar al nivel que ha sido seleccionado.

\section{Resultados}

Durante la última década, varias sociedades internacionales han recomendado fuertemente el protocolo FAST (Grado I) esto debido a que es adecuado para traumatismos abdominales cerrados, traumatismos torácicos cerrados o penetrantes, tanto en pacientes estables como inestables. Como es una prueba dependiente del operador, se requiere la curva de aprendizaje, que se correlaciona con los resultados. "La literatura describe resultados variables según los diferentes operadores (radiólogo versus emergencista), lesiones asociadas del parénquima (para las que no se planificó el método) y estado hemodinámico (hipotensión arterial versus normotensión)" (Gracias \& Frankel, 2001).

Una prueba FAST positiva indica hemorragia en la cavidad y probablemente requiera una laparotomía exploradora si el paciente es hemodinámicamente inestable o un diagnóstico continuo con tomografía computarizada (CT), si está disponible, en pacientes clínicamente estables (Figura 1).

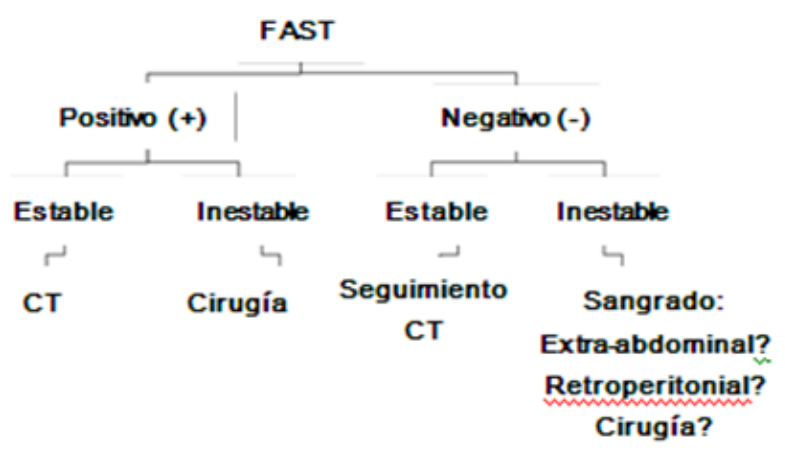

Figura 1. Consenso FAST

Fuente: (Scalea, Rodríguez, \& Chiu, 1999).

Una peculiaridad de FAST es su viabilidad incluso en sitios con métodos complementarios restringidos, como se ve en catástrofes naturales (por ejemplo, terremotos), conflictos bélicos, estaciones espaciales, donde los datos adicionales pueden ser decisivos y no hay otros métodos complementarios disponibles.

El líquido libre aislado en la propia cavidad no implica el requerimiento de cirugía inmediata, ya que debe estar asociado a otros aspectos como la cantidad de líquido libre de la cavidad, el número de sitios (recesos, canalones) con líquido y la clínica del paciente estado.

Algunos autores han propuesto protocolos de puntuación de pacientes FAST positivos, apuntando a la estratificación del riesgo ya sea bajo o alto y la indicación quirúrgica, por ejemplo, la recolección de líquido libre observada en más de tres recesos se correlacionó con un volumen intraperitonial de más de 1000 ml (Sirlin \& Brown, 2005).

La identificación de pacientes de alto riesgo (traumatismo abdominal cerrado e hipertensión arterial) "evidenció la precisión de la prueba en alrededor del 95\%, la sensibilidad del $85 \%$ y la especificidad del $96 \%$, según Lee et al., Para laparotomía exploratoria y consiguiente intervención terapéutica". (McGahan \& Melendres, 2007) 
Con el eFAST "el desarrollo y uso de (Figura 2), la cavidad torácica puede evaluarse simplemente deslizando el transductor cranealmente después de las evaluaciones de Morrison (hepatorrenal) y de los espacios esplenorrenales" (Kirkpatrick, 2007). La secuencia eFAST indica inicialmente la evaluación del abdomen y luego la evaluación del tórax como se describe en la evaluación secundaria de soporte vital avanzado en trauma (evaluación de pies a cabeza), es decir, después de la evaluación primaria.

Sin embargo, la Red Interactiva Mundial Centrada en el Ultrasonido según sus siglas en ingles WINFOCUS (World Interactive Network Focused on Critical Ultrasound) "está desarrollando actualmente el uso del ultrasonido para la evaluación inicial del paciente politraumatizado, desde la evaluación primaria hasta la resolución o el procedimiento quirúrgico. Este protocolo se incluirá en el algoritmo Ultrasound Trauma Life Support (US-ATLS)" (WINFOCUS, 2010). Con base en esta secuencia, se sugiere el abordaje ABC del trauma, comenzando por las vías respiratorias (confirmación de las vías respiratorias permeables y apoyo a la vía aérea quirúrgica), la respiración (neumotórax y hemotórax) y la circulación, por hemorragias como investigación del hemoperitoneo.

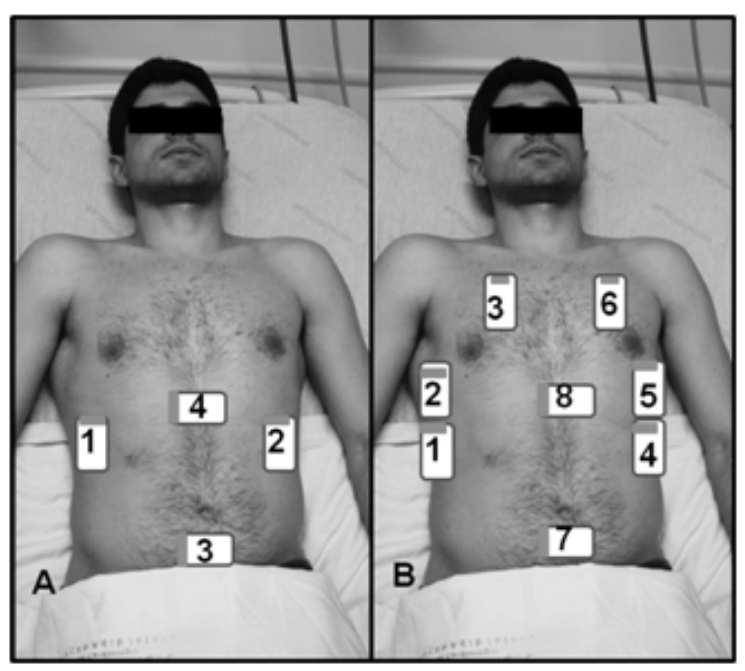

Figura 2. Referencias anatómicas FAST (A) y $\operatorname{EFAST~(B).~}$

Fuente: (Scalea, Rodríguez, \& Chiu, 1999).
En la imagen se observa las referencias anatómicas a considerarse de acuerdo con una evaluación FAST - Evaluación enfocada con ecografía para traumatismos; o una EFAST - Evaluación enfocada extendida con ecografía para traumatismos.

\section{Ecografía pulmonar}

Las referencias anatómicas de tórax correctas como diafragma, parénquima pulmonar, arcos costales y la identificación de artefactos son vitales para una lectura adecuada.

Se pueden diagnosticar cambios como derrame pleural, hemotórax, falta de deslizamiento pleural (neumotórax, intubación selectiva), complicaciones pulmonares relacionadas con el transporte del paciente intubado (como desplazamiento de sonda, entre otros). La relevancia de este método es paralela a la frecuencia de cambios torácicos que se encuentran en pacientes con traumatismos mayores. (WINFOCUS, 2010)

Se evidenció un neumotórax de cada 5 traumatismos mayores que, de no identificarse, podrían conducir a cambios hemodinámicos graves y la muerte. Los autores Blaivas, Lyon, \& Duggal, (2005) "evaluaron la precisión de la radiografía de tórax incidencia anteroposterior versus la ecografía pulmonar para la identificación del neumotórax oculto lectura normal de la radiografía de tórax y neumotórax evidenciado en la TC o la ecografía en pacientes con politraumatismo, y encontraron que esta última tiene una $94 \%$ de precisión en comparación con los rayos X".

El neumotórax oculto por sí solo puede no determinar directamente el deterioro del paciente, sin embargo, cuando se asocia con lesiones secundarias como contusión pulmonar, hipotermia, hipoxia y ventilación con presión positiva, puede tener consecuencias graves. "En estudios observacionales, el neumotórax oculto en pacientes traumatizados se describió mediante TC de tórax en aproximadamente el 55\%" (Kirkpatrick, 2007). Sus posibilidades de identificación 
rápida a pie de cama para realizarse en entornos hospitalarios / o en escenarios de campo de batalla, sin implicar riesgos para el paciente $u$ otros dispositivos de indisponibilidad, pueden optimizar la atención al paciente y, por tanto, reducir la mortalidad.

Esta herramienta tiene como objetivo apoyar el proceso de toma de decisiones del médico tratante y apoyar el seguimiento de las intervenciones farmacológicas y quirúrgicas. La experiencia médica con eFAST US está relacionada con su correcto uso, así como con limitaciones técnicas y errores de lectura de conocimiento adecuado de la anatomía humana. Como este protocolo tiene como objetivo identificar los fluidos libres dentro de las cavidades y el neumotórax, en lugar de evaluaciones de órganos complejos, su curva de aprendizaje es corta y relativamente fácil.

De acuerdo con (Vela, 2018) "la detección de líquido abdominal libre se realiza, según la magnitud, primero en el cuadrante superior derecho (receso hepatorrenal)", que cuando se realiza de forma sistemática toma sólo 19 segundos. Un examen FAST negativo completo dura aproximadamente 3 minutos. Nuevamente, debe enfatizarse que un resultado negativo no excluye lesiones potencialmente mortales, como hemorragias retroperitoniales y lesiones de vísceras huecas, que no están incluidas en el método.

\section{A continuación se presentan las referen- cias de ultrasonido para un eFAST}

- Hepatorrenal rebaje (Figura 3)

- Derecho axilar anterior línea (hígado-pulmón (diafragma) de transición) Figura 4)

- Anterior derecha línea hemiclavicular, entre el $3^{\circ}$ y $5^{\circ}$ espacio intercostal (Figura 5)

- Rebaje esplenorrenal (Figura 6)

- Línea axilar anterior izquierda, transición bazo-pulmón (Figura 7)
- Izquierda anterior línea hemiclavicular entre el $3^{\circ}$ y $5^{\circ}$ espacio intercostal (anterior evaluación hemitórax izquierdo) (Figura 8)

- Espacio retrovesical (espacio Douglas) (Figura 9)

- Ventana pericárdica (aspecto sub-xifoides, 4 cámaras del corazón) (Figura 10)
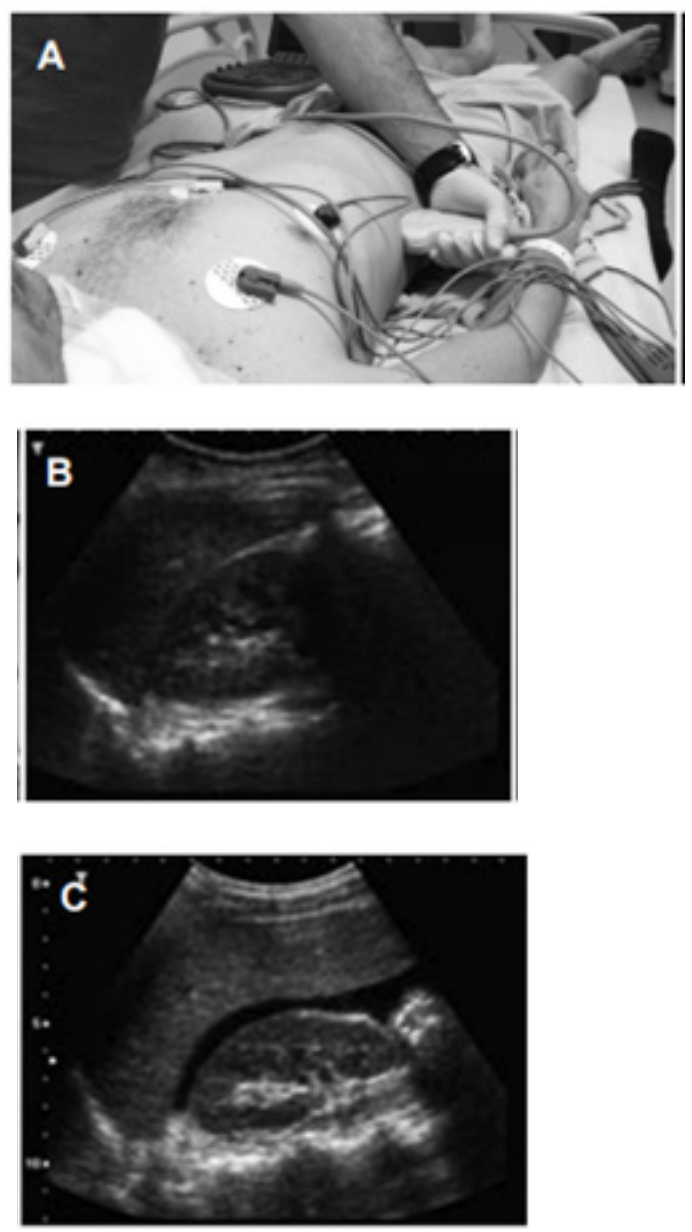

Figura 3. Sonda A-US en el espacio hepatorrenal; B- imagen normal de US.; Cfluido en el espacio de Morrison (FAST +). 

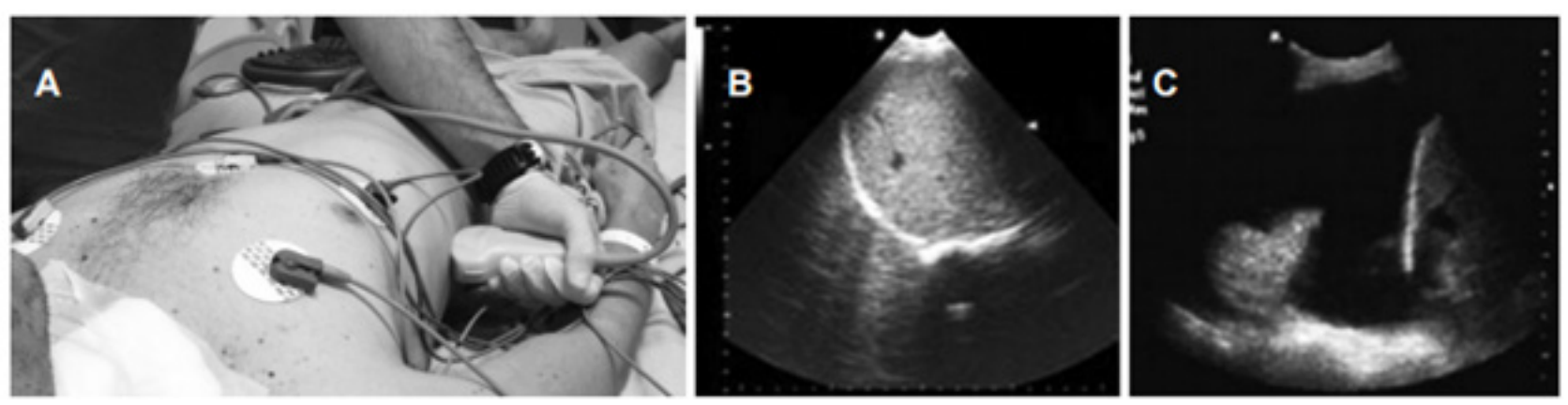

Figura 4. Sonda A- en la intersección diafragma / parénquima pulmonar; B- imagen de la cavidad torácica derecha sin líquido; C- líquido de la cavidad torácica derecha y consolidación pulmonar.
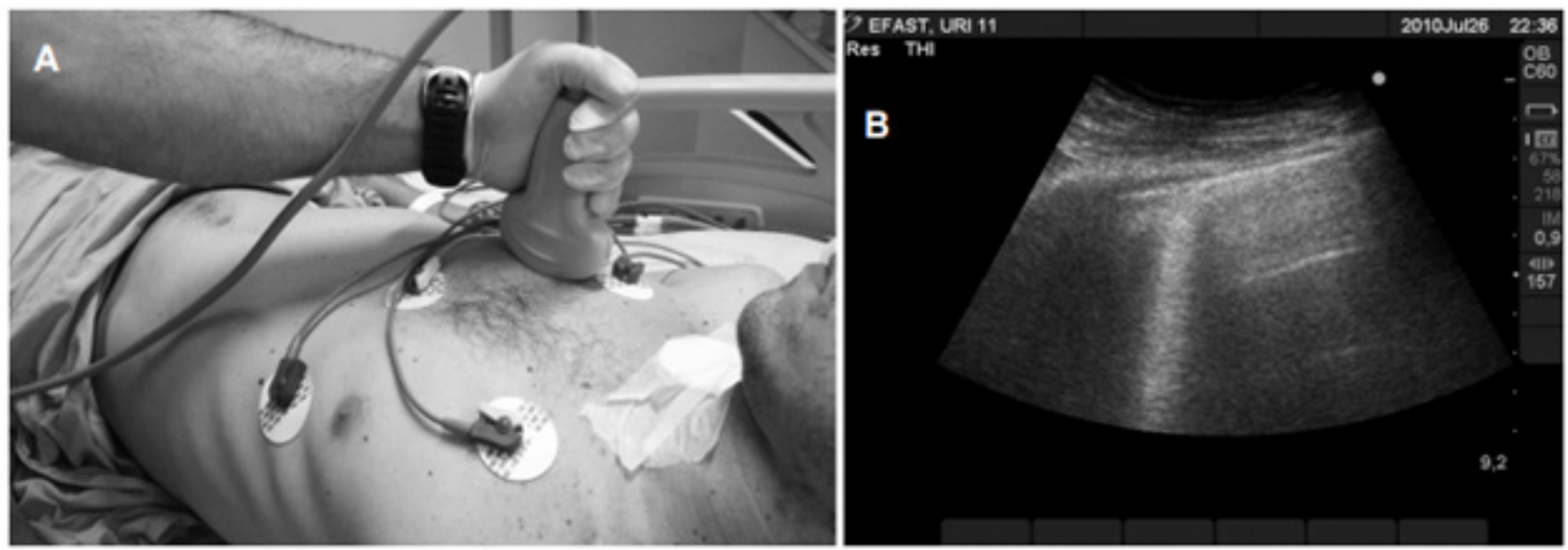

Figura 5. Sonda A- El anterior región del pecho, entre $3^{\circ}$ y $5^{\circ}$ espacio intercostal en la línea hemiclavicular derecho; B- imagen normal de US. (Líneas A y B presentes).
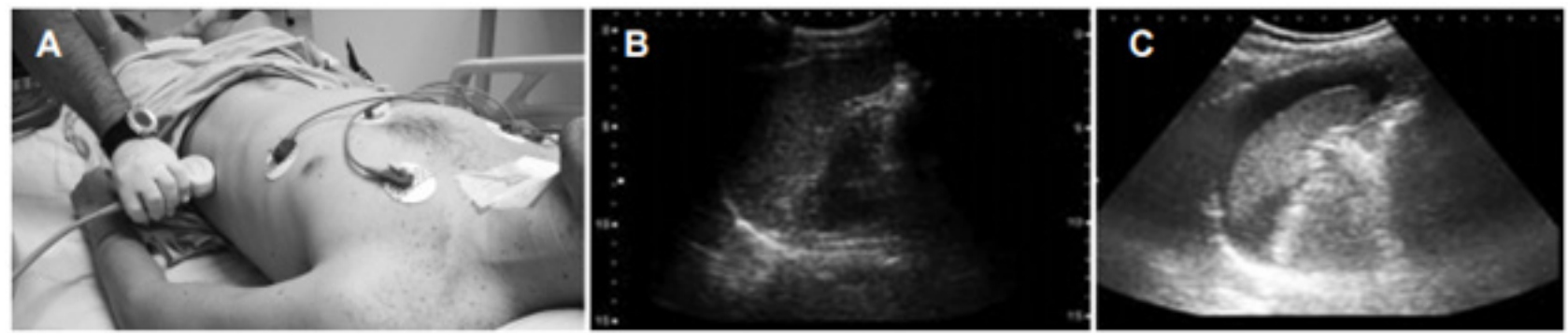

Figura 6. Sonda en el espacio esplenorrenal; B- imagen ecográfica normal; C- líquido del espacio peri-esplénico. 

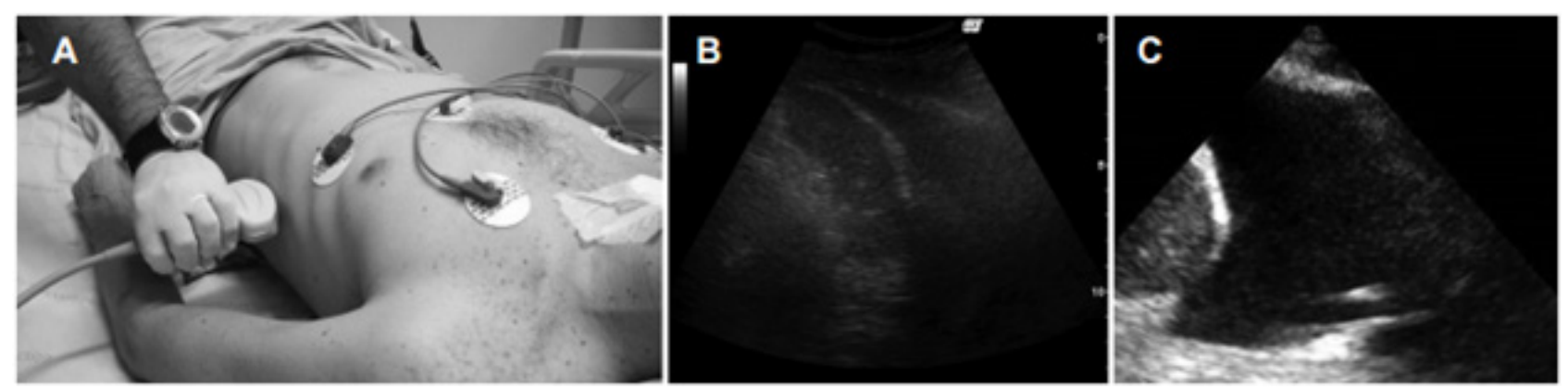

Figura 7. Sonda A en la intersección diafragma izquierdo / parénquima pulmonar; BImagen de la cavidad torácica izquierda sin líquido; C- líquido de la cavidad torácica izquierda (derrame pleural).
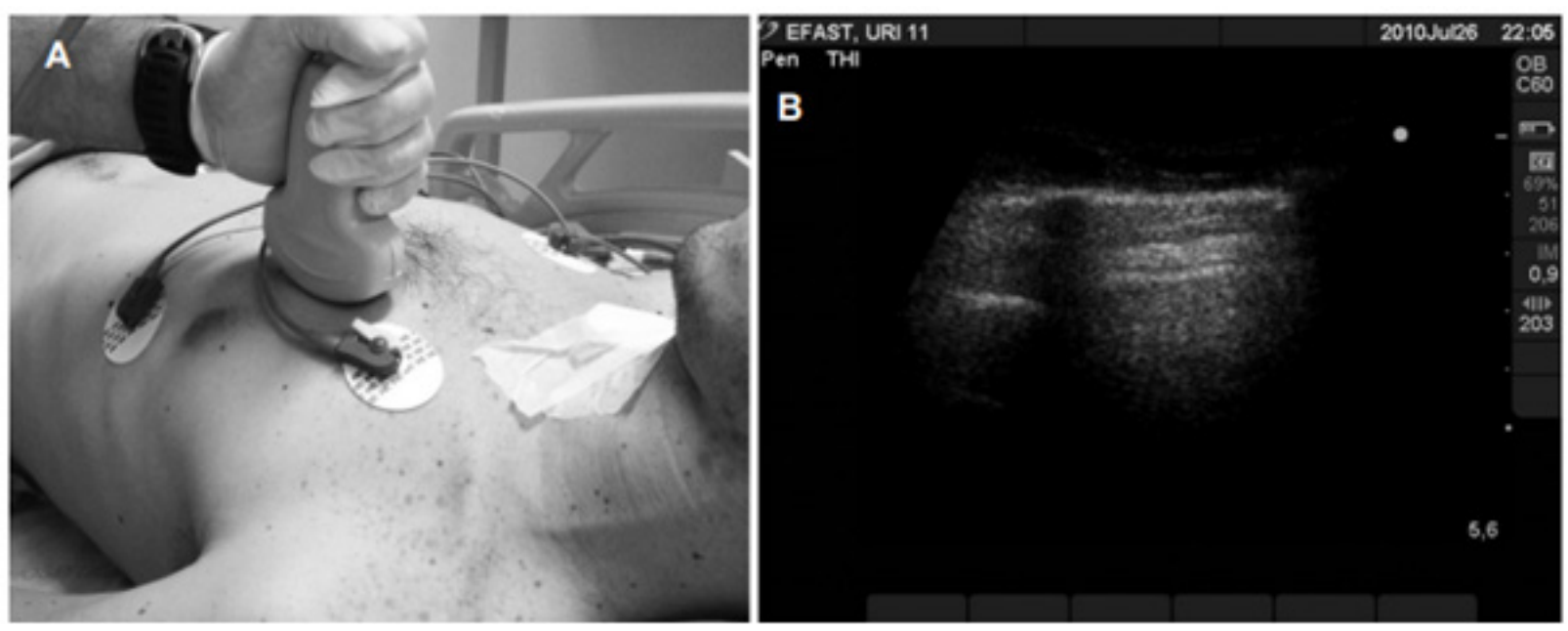

Figura 8. Sonda A- El anterior pecho entre $3^{\circ}$ y $5^{\circ}$ espacio intercostal por la línea hemiclavicular izquierda; B- imagen pulmonar normal
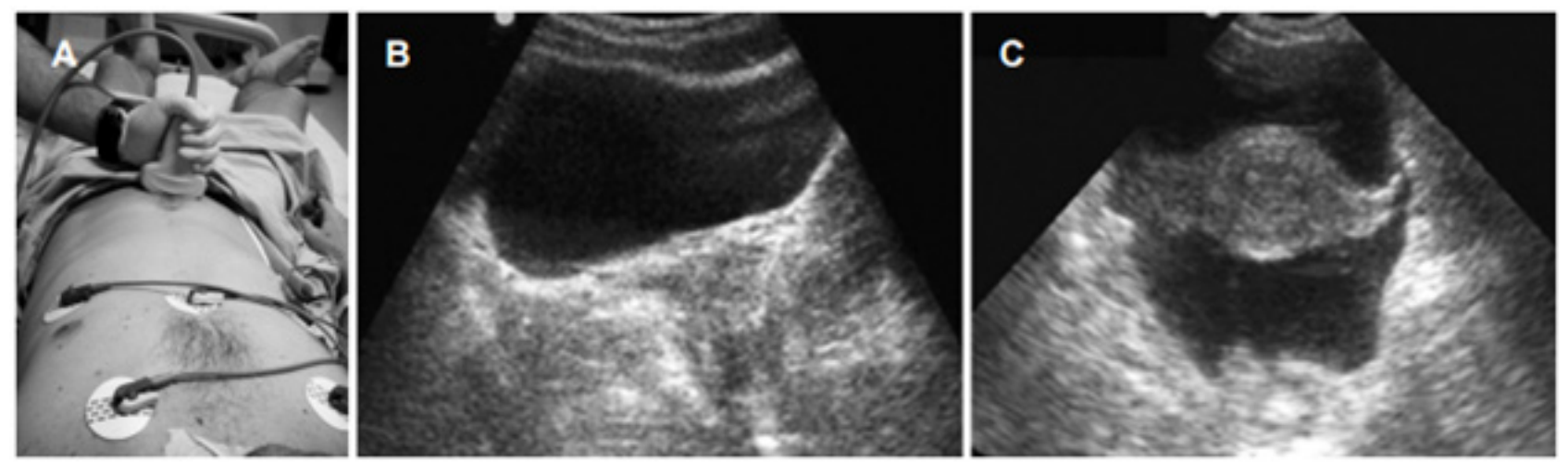

Figura 9. Transductor A- en región hipogástrica; B- vista transversal de un paciente masculino que muestra la vejiga urinaria y el recto; C- líquido libre entre la vejiga urinaria y el recto.

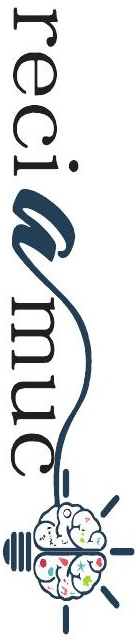


Las complicaciones relacionadas con la TC son un mayor riesgo de cáncer y neprotoxicidad, punto que debe considerarse en el caso de pacientes de edad avanzada. Sin embargo, las nuevas tecnologías portátiles y autosostenibles, como los dispositivos de ultrasonido (US), pueden utilizar la energía solar y transmitirse en tiempo real a los expertos en imagenología. Esta técnica proporciona como ventaja principal su uso en lugares inhóspitos e incluso en unidades de cuidados intensivos, donde el transporte de pacientes no suele ser aconsejable y la agilidad del diagnóstico es un plus.

Su rentabilidad es muy relevante, ya que, algunas pruebas de alto costo utilizadas para el diagnóstico de lesiones agregan costos generales a regiones de recursos muy limitados. La formación y certificación de los profesionales involucrados en la atención de pacientes críticos es probable que se vea en un futuro próximo, ya que esta herramienta está plenamente consolidada entre los médicos de urgencias de países desarrollados desde hace algunos años. Por tanto, el protocolo FAST/eFAST puede ser una gran alternativa con excelentes resultados en el diagnóstico y menos manipulación del paciente.

\section{Bibliografía}

Blaivas, M., Lyon, M., \& Duggal, S. (2005). Una comparación prospectiva de la radiografía de tórax en decúbito supino y la ecografía de cabecera para el diagnóstico de neumotórax traumático. Acad Emerg Med. , 12 (9), 844-9.

Bolívar, J. (2015). Investigación Documental. México. Pax.

Castro, J. (2016). Técnicas Documentales. México. Limusa.

CDC. (2004). Centers for Disease Control and Prevention - CDC. National estimates of the ten leading causes of nonfatal injuries. Centers for Disease Control and Prevention, Atlanta (GA).

Davila, A. (2015). Diccionario de Términos Científicos. . Caracas: Editorial Oasis.

Gracias, V., \& Frankel, H. (2001). Definiendo la curva de aprendizaje para el examen FAST (Sonogram Abdominal Abdominal Sonogram for Trauma). Am Surg. , 67 (4), 364-8.

Kirkpatrick, A. (2007). Clinician-performed focused sonography for the resuscitation of trauma. Crit Care Med , 35 (5), 162-72.

Lichtenstein, D., Pinsky, \& Jardin, F. (2002). General ultrasound in the critically ill. Berlin: Springer.

McGahan, J., \& Melendres, G. (2007). La utilidad de la ecografía para el triaje de pacientes con traumatismo abdominal cerrado a exploratorio por lamparoscopia. AJR Am J Roentgenol. 2007, , 188 (2), 415-21.

Peitzman, B., Billiar, T., Harbrecht, G., \& Kelly, E. (1995). Hemorrhagic shock. Curr Probl Surg. , 32 (11), 925-1002.

Scalea, T., Rodríguez, A., \& Chiu, W. (1999). Evaluación enfocada con ecografía para trauma (FAST). J Trauma , 46 (3), 466-72.

Sirlin, C., \& Brown, M. (2005). Pacientes hipotensos con traumatismo abdominal cerrado: realización de un cribado ecográfico. Radiología. 2005,235 (2), 436-43.

Vela, J. (2018). EFECTO DEL PROGRAMA EDUCATIVO SOBRE EL GRADO DE CONOCIMIENTO DE LA EVALUACIÓN FOCALIZADA CON ECOGRAFÍA PARA TRAUMA (ECOFAST) EN INTERNOS DE MEDICINA HOSPITAL SERGIO E. BERNALES 2016. Lima Peru: Trabajo de Grado.

WINFOCUS. (2010, Jul). World Interactive Network Focused on Critical Ultrasound. (WINFOCUS). UItrasound in critical care medicine - Continuing medical education. . From Available from: http://www. winfocus.org/usccm 


\section{CITAR ESTE ARTICULO:}

Mera Cáceres, M. F., Guachamín Abril, D. K., Paca Ajitimbay, T. N., \& Tatiana Cerón, D. (2020). Valoración mediante ECOFAST en trauma de tórax. RE-

CIAMUC, 4 (4 (esp), 35-46. https://doi.org/10.26820/reciamuc/4.(4).diciembre.2020.35-46

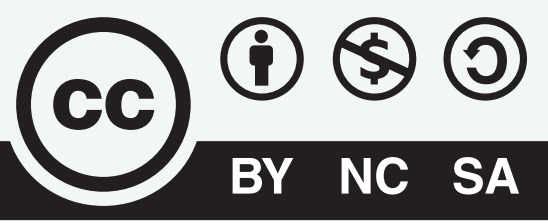

CREATIVE COMMONS RECONOCIMIENTO-NOCOMERCIAL-COMPARTIRIGUAL 4.0 . 